\title{
Association of scores from an online dietary assessment tool with body weight and body composition in the first trimester
}

Maternal body composition and dietary quality in pregnancy have been shown to affect long term health of the offspring ${ }^{(1,2)}$. We have previously shown that scores generated by the dietary assessment tool (DAT) are associated with nutrient intakes which are important to pregnancy outcome ${ }^{(3)}$. This study aimed to determine whether scores generated by the DAT were associated with maternal body weight and body composition in the first trimester.

Women were recruited at their convenience in the first trimester of pregnancy ( $n$ 539). Each participant had their weight and height measured, and underwent bio-electrical impedance analysis (BIA) to assess their body composition. Participants estimated their physical activity level on a six-point scale ${ }^{(4)}$ and completed the online DAT. This online survey elicited summary dietary intake data which was algorithmically evaluated and scored under a number of dietary domains, each representing one aspect of dietary quality. The dietary domain scores were condensed into a single percentage score for overall dietary quality as described previously ${ }^{(3)}$, with higher domain scores and higher overall scores representing more favourable dietary behaviour.

The mean age of the study participants was 30.2 years (SD 5.4 years) and $44.8 \%$ were primiparous. Mean body mass index (BMI) was $25.2 \mathrm{~kg} / \mathrm{m}^{2}\left(\mathrm{SD} 4.8 \mathrm{~kg} / \mathrm{m}^{2}\right)$ and $14.5 \%$ were obese. Higher scores in the breakfast cereal domain were associated with lower BMI $(P=0.01)$, lower percentage body fat $(P=0.02)$, lower fat mass index $(P=0.01)$ and lower waist to height ratio $(P=0.04)$; and with lower fat free mass index $(P=0.04)$. Lower scores in the fatty foods domain were associated with higher BMI $(P<0 \cdot 05)$, higher percentage body fat $(P=0.01)$, higher fat mass index $(P=0.02)$ and higher waist to height ratio $(P=0.02)$. Lower scores in the alcohol domain were associated with greater visceral fat mass $(P=0.04)$. Most notably however, the overall DAT score showed a strong inverse correlation with BMI $(P=0 \cdot 02)$, body fat percentage $(P=0.02)$, waist to height ratio $(P=0 \cdot 01)$ and fat mass index $(P=0 \cdot 01)$.

Scores generated by the DAT are associated with body weight and body composition in the first trimester of pregnancy, with higher dietary quality scores from the DAT associated with more favourable body weight and body composition.

1. Pomeroy E, Wells JC, Cole TJ et al. (2015) Am J Phys Anthropol 156, 625-36.

2. Godfrey KM, Sheppard A, Gluckman PD et al. (2011) Diabetes 60,1528-34.

3. Mullaney L, O'Higgins AC, Cawley S et al. (2016) Use of a web-based dietary assessment tool in early pregnancy. Ir J Med Sci. 2016 Mar 11 - In Press

4. Food and Agricultural Organisation/World Health Organisation/United Nations University (2001) Human energy requirements. Report of a Joint FAO/WHO/UNU Expert Consultation. Rome: Food and Agricultural Organisation. 